\title{
Relationships of gut microbiota, short-chain fatty acids, inflammation, and the gut barrier in Parkinson's disease
}

\author{
Velma T. E. Aho ${ }^{1,2+}$ D, Madelyn C. Houser ${ }^{3,4+}$ (D), Pedro A. B. Pereira ${ }^{1,2}$ (D) Jianjun Chang ${ }^{4}$, Knut Rudi ${ }^{5}$, Lars Paulin $^{1}$ (D),
} Vicki Hertzberg ${ }^{3}$ (D), Petri Auvinen ${ }^{1}$ (D), Malú G. Tansey ${ }^{4,6^{*}}$ (D) and Filip Scheperjans ${ }^{2^{*}}$ (D)

\begin{abstract}
Background: Previous studies have reported that gut microbiota, permeability, short-chain fatty acids (SCFAs), and inflammation are altered in Parkinson's disease (PD), but how these factors are linked and how they contribute to disease processes and symptoms remains uncertain. This study sought to compare and identify associations among these factors in PD patients and controls to elucidate their interrelations and links to clinical manifestations of PD.

Methods: Stool and plasma samples and clinical data were collected from 55 PD patients and 56 controls. Levels of stool SCFAs and stool and plasma inflammatory and permeability markers were compared between patients and controls and related to one another and to the gut microbiota.

Results: Calprotectin was increased and SCFAs decreased in stool in PD in a sex-dependent manner. Inflammatory markers in plasma and stool were neither intercorrelated nor strongly associated with SCFA levels. Age at PD onset was positively correlated with SCFAs and negatively correlated with CXCL8 and IL-1 $\beta$ in stool. Fecal zonulin correlated positively with fecal NGAL and negatively with PD motor and non-motor symptoms. Microbiota diversity and composition were linked to levels of SCFAs, inflammatory factors, and zonulin in stool. Certain relationships differed between patients and controls and by sex.

Conclusions: Intestinal inflammatory responses and reductions in fecal SCFAs occur in PD, are related to the microbiota and to disease onset, and are not reflected in plasma inflammatory profiles. Some of these relationships are distinct in PD and are sex-dependent. This study revealed potential alterations in microbiota-host interactions and links between earlier PD onset and intestinal inflammatory responses and reduced SCFA levels, highlighting candidate molecules and pathways which may contribute to PD pathogenesis and clinical presentation and which warrant further investigation.
\end{abstract}

Keywords: Parkinson's disease, Microbiota, Intestine, Inflammation, Short-chain fatty acids

\footnotetext{
*Correspondence: mgtansey@UFL.edu; filip.scheperjans@hus.fi

${ }^{\dagger}$ Velma T. E. Aho and Madelyn C. Houser contributed equally to this work;

Filip Scheperjans and Malú G. Tansey are co-corresponding authors.

${ }^{4}$ Department of Physiology, Emory University School of Medicine, 615

Michael St, Atlanta, GA 30322, USA

${ }^{2}$ Department of Neurology, Helsinki University Hospital, and Department of

Neurological Sciences (Neurology), University of Helsinki, ward K4A, Haartmaninkatu 4, Fl-00290 Helsinki, Finland

Full list of author information is available at the end of the article
}

\section{Background}

More than thirteen studies from three continents have established that the gut microbial communities of patients with Parkinson's disease (PD) differ from those of healthy subjects [1-3]. Most of these studies have focused on profiling the bacteria present in stool samples but have not linked these findings to functional consequences on microbiome-host interactions, leaving open

C C The Author(s). 2021 Open Access This article is licensed under a Creative Commons Attribution 4.0 International License, which permits use, sharing, adaptation, distribution and reproduction in any medium or format, as long as you give appropriate credit to the original author(s) and the source, provide a link to the Creative Commons licence, and indicate if changes were made. The images or other third party material in this article are included in the article's Creative Commons licence, unless indicated otherwise in a credit line to the material. If material is not included in the article's Creative Commons licence and your intended use is not permitted by statutory regulation or exceeds the permitted use, you will need to obtain permission directly from the copyright holder. To view a copy of this licence, visit http://creativecommons.org/licenses/by/4.0/. The Creative Commons Public Domain Dedication waiver (http://creativecommons.org/publicdomain/zero/1.0/) applies to the data made available in this article, unless otherwise stated in a credit line to the data. 
the questions of whether and how altered bacterial composition could contribute to disease processes in PD. Microbially-produced short-chain fatty acids (SCFAs) and regulation of immune responses and gut permeability are some of the proposed mechanisms by which gut microbes could impact brain health and function $[4,5]$.

SCFAs, particularly acetic, propionic, and butyric acid, are the main end product of bacterial fiber fermentation in the gut [6]. They have many effects on host physiology including acting as an energy source for colonocytes $[7,8]$, regulating the gut barrier [9], and influencing inflammatory responses [10]. SCFAs have been suggested as key mediators in microbiota-gut-brain interactions [11]. In a study contrasting fecal SCFA levels in PD patients and control subjects, decreased concentrations of SCFAs, particularly butyric acid, in PD patients were linked to microbiota alterations [12], and several bacterial taxa reportedly reduced in PD are SCFA producers $[2,3,13]$.

Inflammation is a widely recognized hallmark of PD. Increased levels of a variety of inflammatory molecules in brain and cerebrospinal fluid (CSF) [14-16] as well as blood [17-19] from PD patients have been documented. While the same specific cytokines, chemokines, and other inflammatory markers are not consistently implicated, IL6, TNF, IL-1 $\beta$, CRP, IL-10, CCL5, and IL-2 are among the most commonly dysregulated in the peripheral blood of PD patients [18]. It has been suggested that this inflammation may result from intestinal barrier deficiency which could increase systemic exposure to inflammatory microbial products such as lipopolysaccharide (LPS), a component of bacterial cell walls [5, 20]. Experiments measuring excretion of ingested sugars suggest increased permeability of the colon (but not the small intestine) in PD [2123]. Higher levels of zonulin and alpha-1-antitrypsin, indicators of gut permeability, have been found in PD patients' stool relative to controls [24], although this difference has not been consistently observed [25]. Additionally, increased detection of LPS [26] and decreased LPS-binding protein (LBP) $[21,27]$ in blood and plasma from PD patients suggest greater exposure of peripheral tissues and immune cells to LPS, supporting the existence of PDrelated gut barrier dysfunction.

In recent years, there has been increasing recognition of a low-grade inflammatory state in the gut in PD. Studies have reported increased expression of genes encoding proinflammatory cytokines and chemokines in gut tissue from PD patients compared to controls [22, 28 ], and higher levels of IL-1 $\alpha$, IL-1 $\beta$, CXCL8 (also known as IL-8), and CRP have been found in stool [29]. The neutrophil-associated protein calprotectin is an indicator of gut inflammation in inflammatory bowel disease [30], and increased levels of calprotectin have been found in the stool of PD patients relative to controls [24,
25]. Finally, higher numbers of CD3+ T cells and cells expressing the LPS receptor Toll-like receptor 4 (TLR4) have been identified in colon tissue from PD patients. When TLR4 was knocked out in a mouse model of PD pathology, symptoms were mitigated [22].

Taken together, these findings support the hypothesis that intestinal bacteria and their metabolites along with inflammation and barrier dysfunction impact PD pathophysiology. While there have been several studies evaluating inflammatory and gut permeability markers in PD, this study addresses the gap in knowledge regarding the interplay of these molecules in the blood and the gut and with the gut microbiota and SCFAs in PD patients and healthy control subjects. Identifying how these factors relate to one another and to the onset and clinical presentation of PD is a key step in elucidating the mechanisms behind these disease processes and pinpointing candidate molecules and pathways to target for preventive and therapeutic intervention.

\section{Methods}

Additional details are available in Additional File 1.

\section{Study subjects and sampling}

The study subjects were originally recruited for a pilot study of gut microbiota and PD [31]. Samples for the present study were collected at a follow-up timepoint together with extensive clinical data, including questionnaires on diet, non-motor symptoms, and PD severity; full details have been published previously [32]. The majority of the patients in this study were classified as being in the early to mid-stages of PD (2-3 on Hoehn and Yahr scale). Subjects collected stool samples at home into tubes with DNA stabilizer for microbiota analyses and tubes without preservatives for SCFA and immunological analyses. They kept the samples refrigerated until transport to the clinic (up to 3 days) for storage at $-80^{\circ} \mathrm{C}$. Venous blood samples were collected into EDTA tubes, centrifuged (10 min, $3000 \mathrm{rpm})$, and plasma was divided into aliquots and stored at $-80^{\circ} \mathrm{C}$. Samples were distributed to the laboratories by overnight shipping on dry ice. After excluding subjects with missing values for microbiota, acetic, butyric, or propionic acid, or most immune markers, the total number of subjects remaining for analysis was 111 (55 patients, 56 control subjects).

\section{Microbiota data}

The microbiota data analyzed in this study have been published previously with a detailed description of the workflow [32]. Briefly, stool samples were collected into PSP Spin Stool DNA Plus Kit tubes (STRATEC Molecular). DNA was extracted with the corresponding kit from the same manufacturer. The V3-V4 regions of the $16 \mathrm{~S}$ 
rRNA gene were PCR-amplified and sequenced with Illumina MiSeq. Sequence quality control, Operational Taxonomic Unit (OTU) clustering, and taxonomical classification were performed with mothur [33, 34]. Enterotypes were determined with an online tool [35, 36].

\section{Short-chain fatty acid measurements}

Detection and quantification of SCFAs in the stool samples were performed at the Norwegian University of Life Sciences using a Trace 1310 gas chromatograph (Thermo Fisher Scientific).

\section{Inflammatory and permeability marker measurements}

Factors of interest in stool were measured using the Zonulin Stool ELISA (ALPCO 30-ZONHU-E01), LEGE ND MAX $^{\mathrm{sm}}$ Human MRP8/14 (Calprotectin) ELISA (Biolegend 439,707), LEGEND MAX ${ }^{\mathrm{ma}}$ Human NGAL (Lipocalin-2) ELISA (Biolegend 443,407), and the V-PLEX Proinflammatory Panel 1 Human (Meso Scale Discovery [MSD], Rockville, MD, K15049D) kits. The V-PLEX kit was also used to measure inflammatory mediators in plasma, as was the Human LBP Kit (MSD K151IYC). Stool and plasma samples were prepared, assays run, and results analyzed according to manufacturers' protocols by the Emory Multiplexed Immunoassay Core.

\section{Statistical analyses}

All statistical analyses were performed in $\mathrm{R}$ [37] with packages including phyloseq [38], vegan [39], and DESeq2 [40] for microbial data comparisons. For statistical comparisons between SCFAs/markers, clinical variables, enterotypes, and alpha diversity, we used the Kruskal-Wallis test, Wilcoxon rank sum test, or Pearson correlations depending on the types of variables. When multiple comparison corrections were included, we used the Benjamini \& Hochberg false discovery rate (FDR), correcting SCFAs, stool markers, and plasma markers separately. Intercorrelated inflammatory markers were merged based on Principal Component Analysis (PCA). The complete analysis is included as Additional File 2.

\section{Results}

PD patients have higher calprotectin and lower SCFA levels in stool and lower CXCL8 levels in plasma compared to controls

Patient and control groups were similar with regard to basic demographics such as age, sex, and body mass index (BMI); however, as we knew from previous analyses of the same subjects [31,32], the groups differed regarding medications, medical history, and various symptom scores. A higher percentage of controls reported a history of stroke and use of medications for high blood pressure and cholesterol, and PD patients scored higher on scales of non-motor symptoms, gastrointestinal problems, and constipation (Table 1). Contrasting inflammatory markers and SCFAs between PD patients and control subjects, patients had lower levels of butyric and propionic acid and higher levels of calprotectin in their stool and lower levels of CXCL8 in plasma (Additional File 3A). When the data were stratified by sex, the differences were particularly prominent for butyric acid in males and for plasma CXCL8 and stool calprotectin in females (Fig. 1, Additional File 3B). For calprotectin, this sex effect may be explained in part by relative baseline levels of the protein. Among control subjects, calprotectin levels trended lower in females than in males $(p=0.051)$ (Additional File 3B).

\section{Inflammatory markers in plasma and stool are not correlated, but stool SCFAs, zonulin, and stool and plasma cytokines are related to PD onset and symptom severity}

The inflammatory markers measured from the same sample material (plasma or stool) were largely intercorrelated, but we found no correlations between measurements of the same markers in stool and plasma (Fig. 2). PCAs for combining the most correlated markers resulted in one principal component (PC) for stool (summarizing IL-10, IL-12p70, IL-13, TNF, IL-6, and IFN $)$, and two plasma PCs (PC1: IL-10, IL-4, TNF; PC2: IL-1 $\beta$, IL-2, IL-12p70, IL-13) (Additional File 2). In stool, the inflammatory markers neutrophil gelatinase-associated lipocalin (NGAL) and calprotectin and the gut permeability marker zonulin were significantly correlated (Fig. 2). In general, calprotectin and zonulin showed fewer correlations with other stool markers in the PD group as compared to the control group. Except for correlations with stool CXCL8 and IL-1 $\beta$ in the control group, none of the three most abundant SCFAs was significantly correlated with any of the markers in stool or plasma (Fig. 2).

Contrasting clinical variables against SCFAs and inflammatory markers suggested inverse correlations between stool SCFAs and several PD-related clinical variables such as the Non-Motor Symptoms Scale (NMSS) total score (which assesses factors including orthostatic hypotension, anosmia, depression, anxiety, cognitive impairment, constipation, and abnormalities in sleep, perception, and urinary patterns), the Rome III constipation/defecation subscore, stool consistency associated with constipation on the Victoria Bowel Performance Scale (BPS), and the Geriatric Depression Scale-15 (GDS15) (Fig. 3a, Additional File 4A). A similar pattern was seen for the Rome III constipation/defecation subscore, Rome III irritable bowel syndrome (IBS) criteria, and NMSS score and plasma CXCL8 (Fig. 3b, Additional File 4A). These correlations were significant for the entire cohort, and some were significant in controls, 
Table 1 Demographic and Clinical Details of Subjects

\begin{tabular}{llll}
\hline & Control subjects & PD patients & $p$-value \\
\hline Number of Subjects & 56 & 55 & 52.73 \\
Sex (\% Male) & 48.21 & $67.63 \pm 5.21$ \\
Age at stool collection (mean \pm SD) & $66.38 \pm 6.73$ & $27.63 \pm 4.74$ \\
Body Mass Index (mean \pm SD) & $26.7 \pm 3.56$ & 5.56 & 0.706 \\
History of TIA/ischemic stroke (\%) & 37.50 & 29.09 \\
Medication: ACE-inhibitor or AT1 antagonist (\% yes) & 48.21 & 5.45 & 0.293 \\
Medication: calcium channel blocker (\% yes) & 19.64 & 18.18 \\
Medication: statin (\% yes) & 48.21 & $608.61 \pm 301.46$ \\
Levodopa equivalent daily dose (LEDD mg; mean \pm SD) & $0 \pm 0$ & $48.36 \pm 36.55$ \\
Non-Motor Symptoms Scale (NMSS) total (mean \pm SD) & $7.09 \pm 6.35$ & $7.45 \pm 5.22$ & 0.051 \\
Rome III constipation subscore (items 9-15; mean \pm SD) & $2.46 \pm 3.16$ & 36.36 & 0.042 \\
Rome III irritable bowel syndrome criteria fulfilled (\%) & 7.14 & 0.001 \\
\hline
\end{tabular}

p-values reflect Fisher's exact test, $S D$ standard deviation, TIA transient ischemic attack, ACE Angiotensin-Converting Enzyme, AT1 angiotensin II type 1.

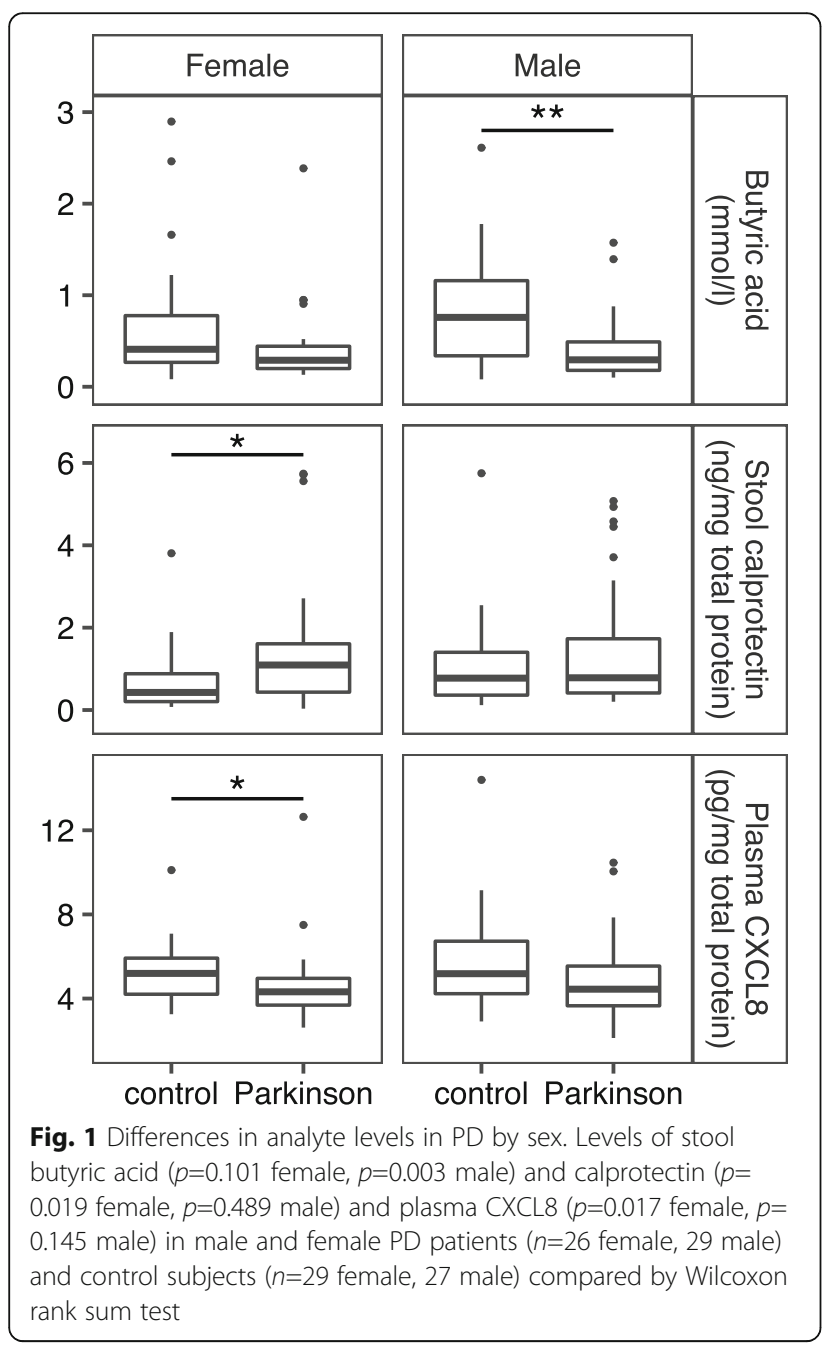

but not when evaluated only within PD patients. Butyric acid levels were correlated, however, with the age of onset for motor and non-motor symptoms of PD, while CXCL8 and IL-1 $\beta$ levels in stool were inversely correlated with the age of motor symptom onset (Fig. 3a, c, Additional File 4B). Acetic acid, while not significantly associated with constipation-related stool consistency in PD patients, was positively correlated with defecation frequency (Fig. 3a). Higher levels of CXCL8 in stool were also associated with constipation-related stool consistency in PD patients and with lower defecation frequency in controls (Fig. 3c). In PD patients, calprotectin levels were inversely linked to IBS symptoms, and stool zonulin was inversely correlated with variables related to PD severity, including the Unified Parkinson's Disease Rating Scale (UPDRS) score, Hoehn and Yahr, GDS15, and NMSS score (Fig. 3c, Additional File 4B). Symptom onset- and severity-related correlations remained significant after correcting for disease duration (Additional File 2).

\section{Microbial alpha and beta diversity are associated with severity of PD symptoms}

In PD patients, several measures of symptom severity were positively correlated with microbial alpha diversity indices, which indicate richness and evenness of bacterial taxa. These included UPDRS scores, Hoehn and Yahr score, and subscales assessing tremor, rigidity, and postural instability and gait difficulty (PIGD) (Additional File 5A, 5B). Microbial beta diversity describes the degree to which different communities of bacteria differ from one another in composition. We found that beta diversity was also significantly associated with UPDRS scores, rigidity, and PIGD. Additionally, the usage of PD medications was significantly related to beta diversity (Additional File 5C). 
A. Full data

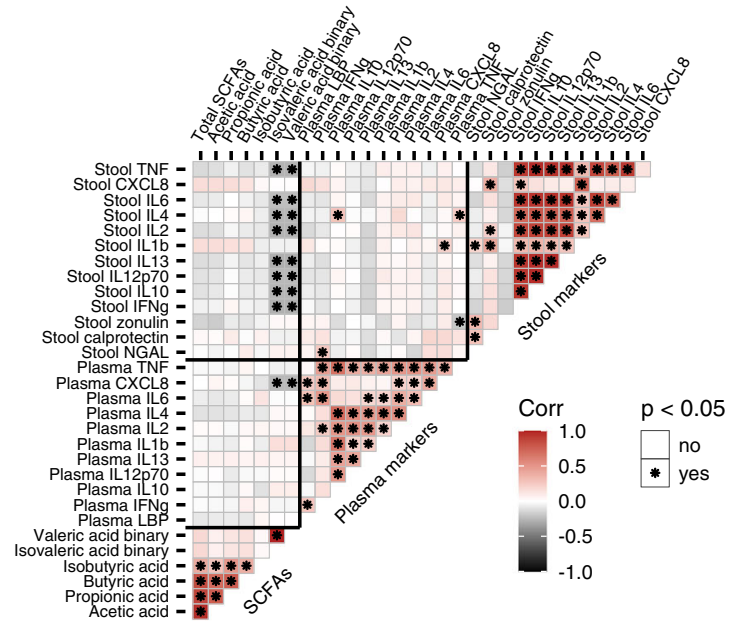

B. Control only

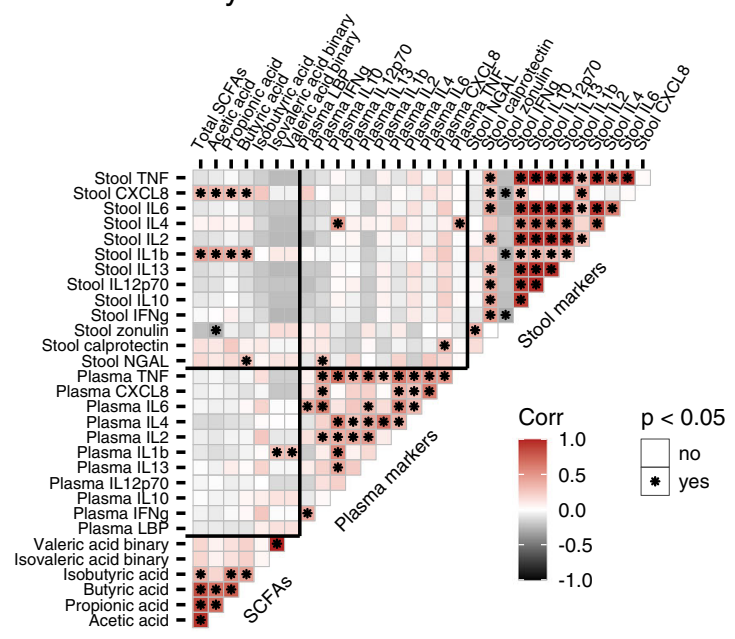

C. PD only

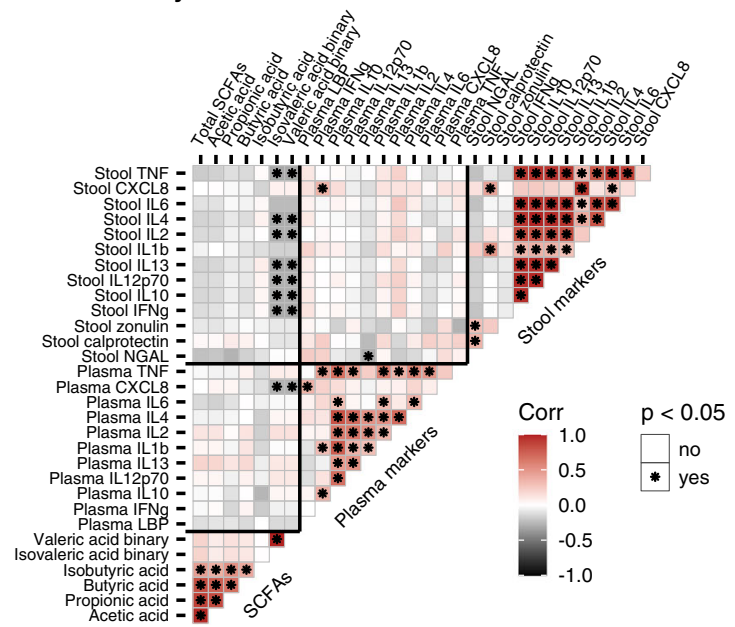

Fig. 2 Correlations among SCFAs and inflammatory and permeability markers. Pearson correlations in a full data, b Control subjects $(n=56)$, and c PD patients $(n=55)$
Microbial alpha diversity is inversely associated with stool inflammatory and permeability markers and SCFAs

Microbial alpha diversity indices were inversely correlated with total SCFAs, acetic, butyric, and propionic acid, and NGAL in stool in control subjects but not in PD patients (Fig. 4). In patients only, a significant inverse correlation between the Shannon diversity index and stool CXCL8 was found (Fig. 4). Inverse correlations between alpha diversity indices and stool calprotectin, zonulin, and IL-1 $\beta$ were significant in the whole cohort, but when the cohort was split by PD status, only the correlations between the Shannon diversity index and calprotectin in PD patients and between the Shannon diversity index and zonulin in control subjects remained statistically significant.

Linear regression modeling corrected for sex and $\mathrm{PD} /$ control status suggested that the relationship between alpha diversity and stool inflammatory markers was independent of sex except for calprotectin, for which an inverse relationship was observed in females but not in males (Fig. 6a, Additional File $6 \mathrm{~A}-\mathrm{C})$. There was a significant interaction between $\mathrm{PD} /$ control status and alpha diversity for propionic acid, showing an inverse correlation between microbial alpha diversity and propionic acid concentration in controls but not in PD patients (Fig. 5b). The total SCFAs variable showed a similar trend (Additional File 6B). The interaction in the propionic acid model remained significant after confounder correction (Additional File 6D).

Microbial beta diversity is related to SCFA levels and inflammatory and permeability markers in stool

We found significant associations between beta diversity and total SCFAs, acetic, butyric, and propionic acid as well as stool NGAL, zonulin, IL2, and the stool marker PC in full data, with or without correction for $\mathrm{PD} /$ control status, sex, constipation, and BMI (Additional File 7A). The effect of calprotectin, while not statistically significant, was worth noting ( $p=0.058$ for full data, $p=0.079$ when corrected for confounders). Control-only comparisons showed significant effects for SCFAs, NGAL, and zonulin; only butyric acid and zonulin were significant in PD-only comparisons (Additional File 7A, 8). These two variables also had a significant interaction with $\mathrm{PD} /$ control status when tested with the variable split into two categories by median (Additional File 7B).

The Prevotella enterotype is associated with higher levels of butyric acid and lower levels of NGAL and zonulin in stool

One broad characterization method for microbiota composition is defining enterotypes based on certain indicator species in bacterial communities [36]. In our subjects, the Firmicutes enterotype was more common and the Prevotella enterotype less common among PD patients compared to control subjects (Additional File 9A). Stool zonulin, NGAL, 

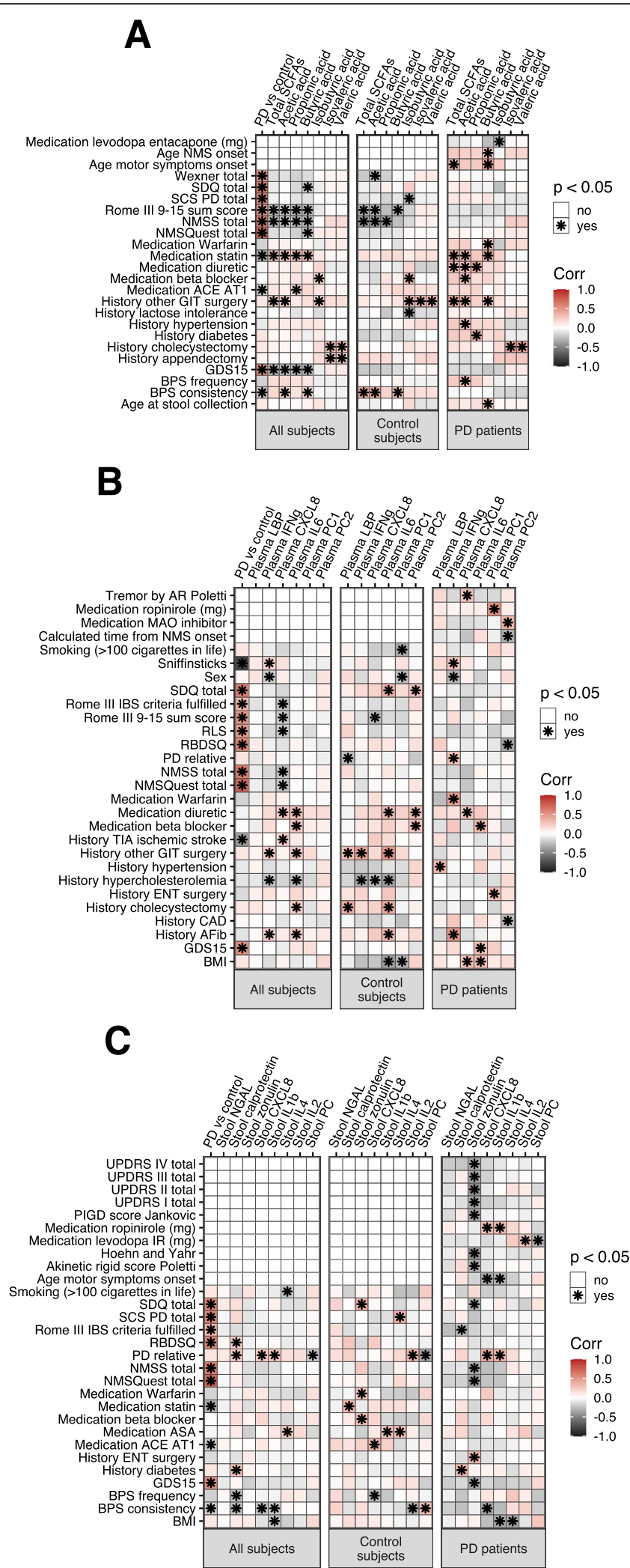

Fig. 3 Correlations of SCFAs, immune markers, and clinical variables. Pearson correlations of a SCFAs, and immune markers in $\mathbf{b}$ plasma and c stool with clinical variables in full data and in only control subjects $(n=56)$ or PD patients $(n=55)$ 


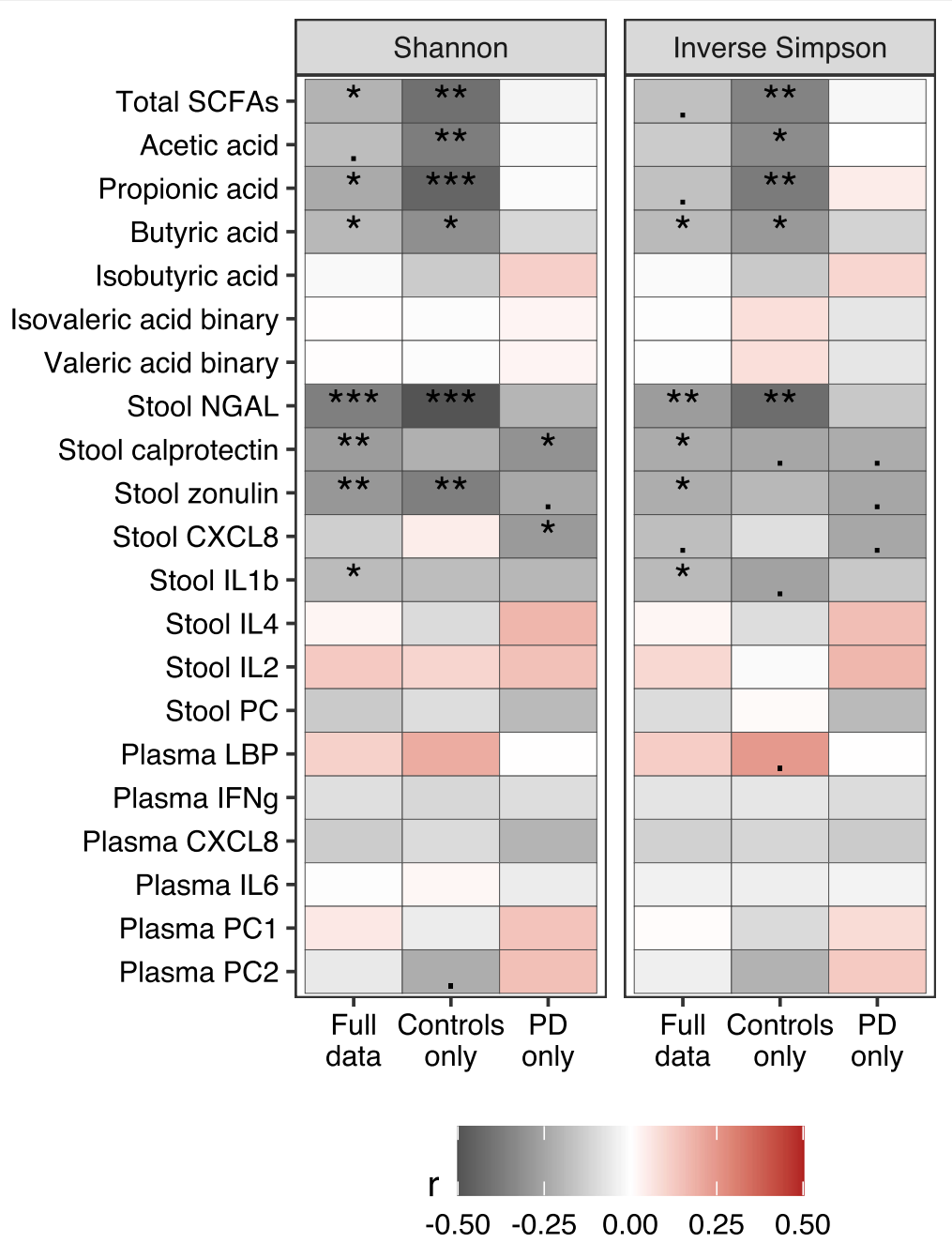

Fig. 4 Correlations of alpha diversity for SCFAs and inflammatory and permeability markers. Pearson correlations with Shannon and inverse Simpson diversity indices, $\mathrm{n}=56$ controls, $n=55$ PD patients; $p$-value is marked as follows: ***: $p \leq 0.001 /{ }^{* *}: p \leq 0.01 /^{*}: p \leq 0.05 / .: 0.1>$ $p>0.05$

propionic acid, and butyric acid had differences in concentrations between enterotypes (Additional File 9B). The difference in propionic acid was significant only in all subjects, not in controls or PD patients separately. Butyric acid levels were higher in PD patients with the Prevotella enterotype than the other two enterotypes, and control subjects with this enterotype had the lowest concentrations of NGAL and zonulin (Fig. 6). The findings regarding NGAL (full data and controls) and zonulin (controls only) remained significant after FDR correction (Additional File 9B).

Specific bacterial taxa are associated with levels of stool SCFAs and inflammatory and permeability markers

Guided by the associations with beta diversity, we explored the associations of specific bacterial taxa with the three most abundant SCFAs and with stool NGAL, calprotectin, and zonulin. The abundances of Butyricicoccus, Clostridium sensu stricto, and Roseburia were positively correlated with levels of SCFAs while the abundances of Akkermansia, Escherichia/Shigella, Flavonifractor, Intestinimonas, Phascolarctobacterium, and Sporobacter decreased with increasing SCFA concentrations (Fig. 7, Additional File 10, 11A). Similar patterns could be seen for the parent families of these taxa, such as a positive association between butyric acid and Lachnospiraceae and a negative one for Enterobacteriaceae and Verrucomicrobiaceae (Additional File 11A). A positive association between SCFAs and Bacteroides was observed only in controls, while a negative association with Bifidobacterium was observed only in PD patients (Fig. 7, Additional File 10, 11A).

Microbiota associations with the stool markers differed between PD patients and control subjects. Stool NGAL levels were positively associated with Bifidobacterium in PD but with Bacteroides and 

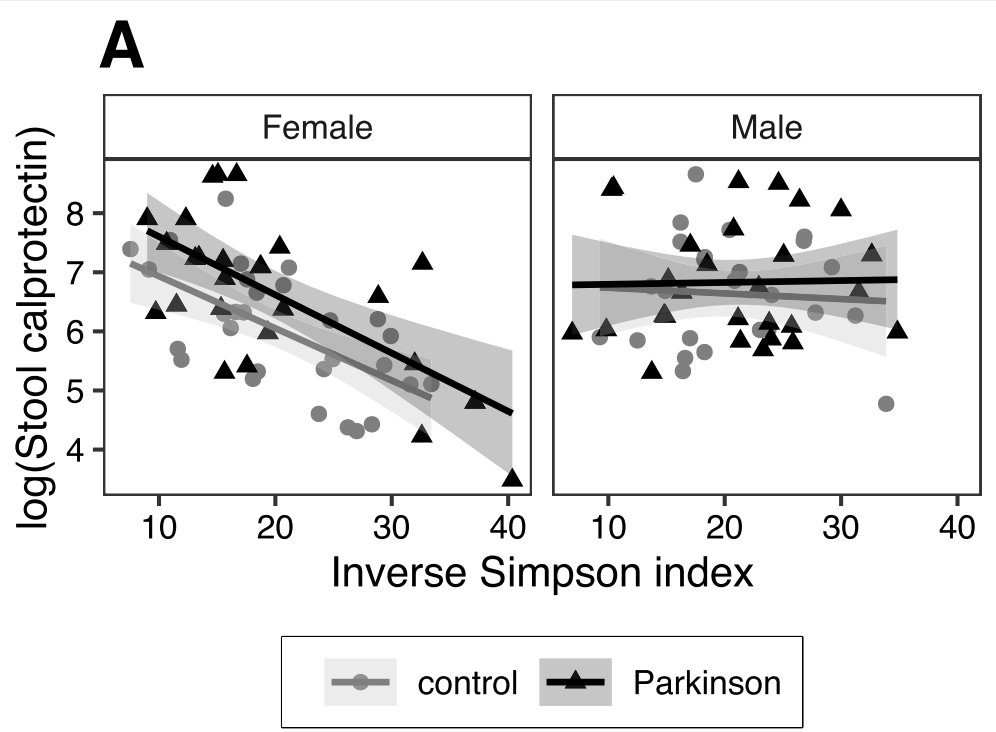

B
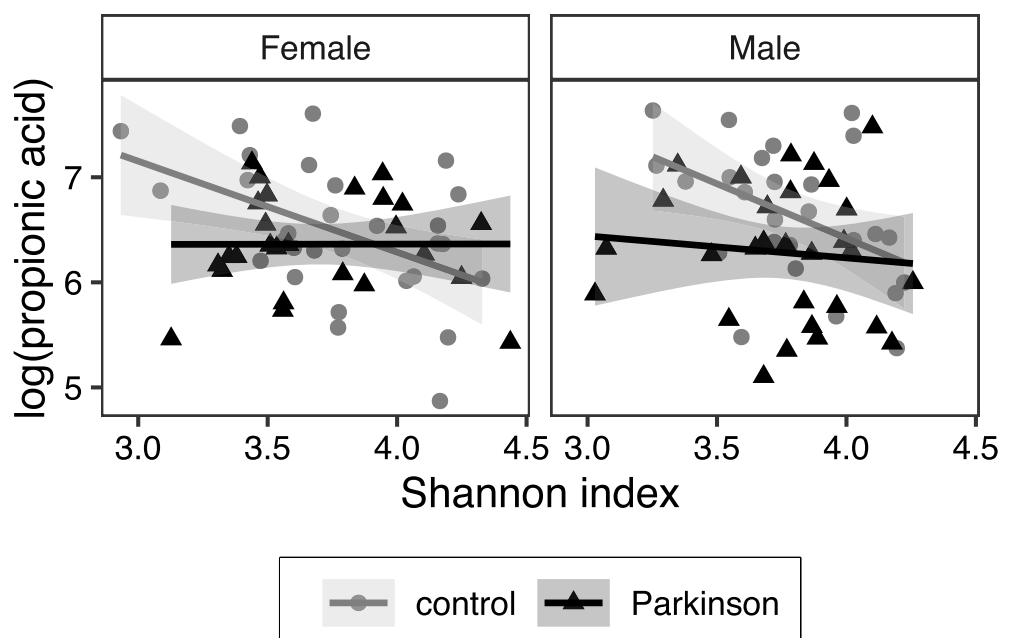

Fig. 5 Linear modeling for alpha diversity showing effects of PD/control status and sex. a Stool calprotectin and inverse Simpson index and $\mathbf{b}$ propionic acid and Shannon index for male and female PD patients ( $n=26$ female, 29 male) and control subjects ( $n=29$ female, 27 male)

Roseburia in controls (Fig. 7, Additional File 11B, 12A). Relative abundance of Prevotella was negatively associated with both NGAL and zonulin in controls only (Fig. 7, Additional File 11B, 12). The only microbiota-immune association found to be significant in both PD patients and controls was the negative relationship between stool zonulin and the genus Coprococcus (Fig. 7, Additional File 11B, 12B), a taxon known to have a strong influence on gut permeability [41] and stool zonulin levels [42]. Other associations with low-abundance taxa were statistically significant but were driven by a small number of data points, precluding confident interpretation (Additional File 12).
Key findings from this study are summarized in Additional File 13.

\section{Discussion}

Numerous studies have reported abnormalities in the gut microbiome, levels of SCFAs, peripheral immune responses, and intestinal permeability in PD; however, this study provides for the first time an integrative analysis of these factors in the same subjects, allowing an evaluation of whether and how they are related to one another and to clinical manifestations of PD.

Efforts have been made to identify biomarkers of PD in both blood and stool, but the relationship between the levels of inflammatory markers in the gut and in 


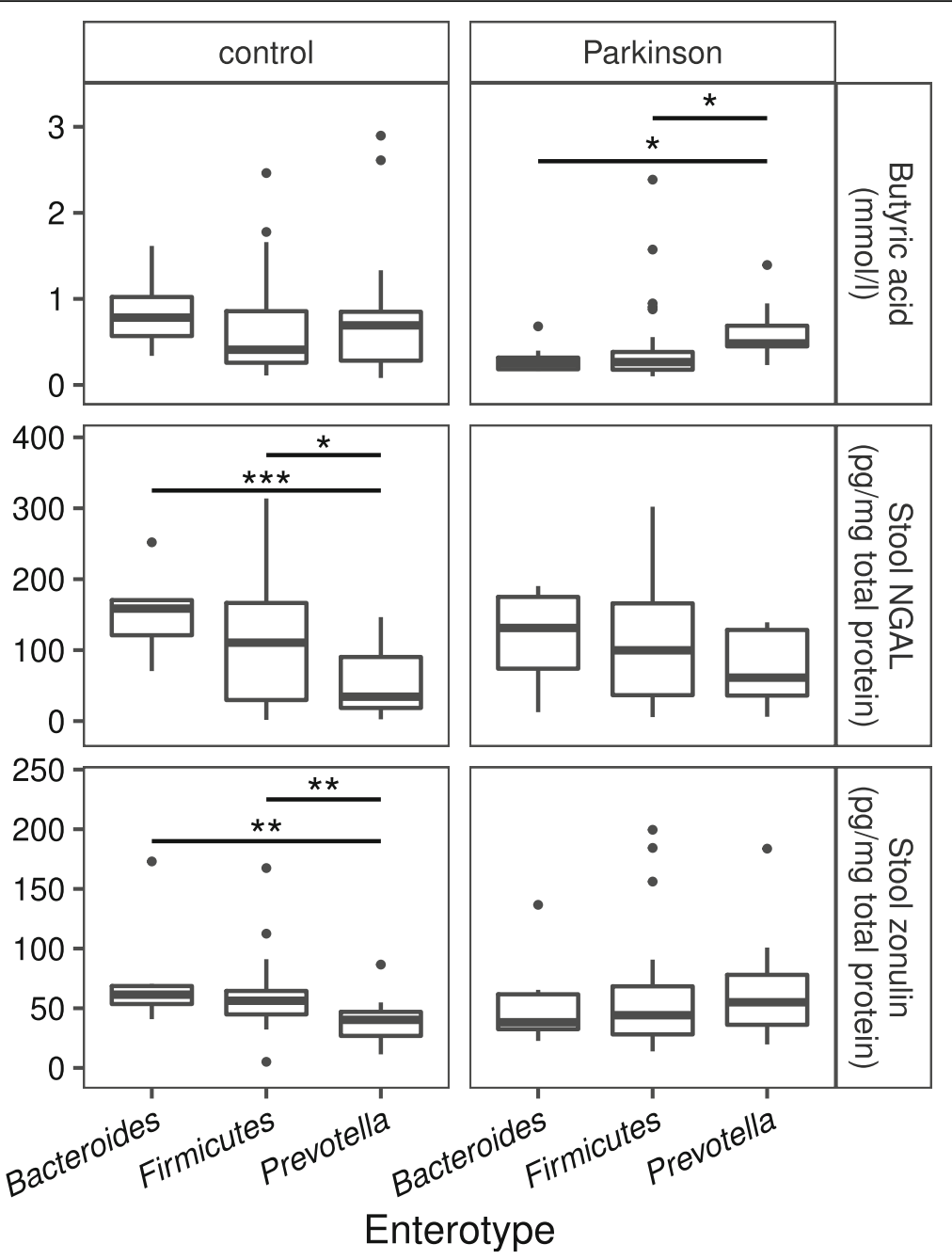

Fig. 6 Butyric acid, NGAL, and zonulin levels by enterotype among PD patients and control subjects. Levels of butyric acid ( $p=0.013$ for PD patients), stool NGAL ( $p=0.002$ for control subjects), and stool zonulin ( $p<0.001$ for control subjects) compared across enterotypes by KruskalWallis test. Asterisks indicate results of pairwise Wilcoxon rank sum post-hoc tests (Bacteroides - 8 control, 8 PD; Firmicutes -25 control, 35 PD; Prevotella - 22 control, 10 PD) with ***: $p \leq 0.001 /^{* *}: p \leq 0.01 / *: p \leq 0.05$

circulation in this disorder had not been elucidated. We determined that levels of inflammation-associated molecules in the plasma and in the stool were highly correlated within each sample type. We found essentially no correlation, however, between levels of inflammatory markers in plasma and stool. This indicates that systemic and gut immune responses are distinct and, thus, measurements from one site are unlikely to represent the nature of the responses in the other.

In the plasma, we found a significant decrease in levels of the chemokine CXCL8 (also known as IL-8) in female PD patients as well as an inverse correlation between CXCL8 and non-motor symptom severity scores in the full cohort. CXCL8 is the primary chemoattractant for neutrophils and is commonly produced in inflammatory responses to stimulate and recruit these phagocytic cells. A previous study has reported a positive correlation between CXCL8 levels in the serum and the degree of PD-related disability [43], rendering our findings somewhat unexpected. A possible explanation is that plasma CXCL8 levels in this cohort are significantly higher in subjects with a history of stroke (Fig. 3), and there are more of these individuals in the control than PD group.

In stool, we documented lower levels of SCFAs and higher levels of calprotectin in PD patients. This is consistent with other reports of SCFA and SCFA-producing bacteria reductions $[3,12,13,19,22,31]$ as well as increased stool calprotectin $[24,25]$ in PD. While SCFAs and calprotectin are beginning to emerge as some of the most reliable indicators of a dysregulated and inflammatory gut environment in $\mathrm{PD}$, our results suggest that these associations may be more pronounced in males and in females, respectively. Levels of butyric acid specifically were correlated with the age of onset for 


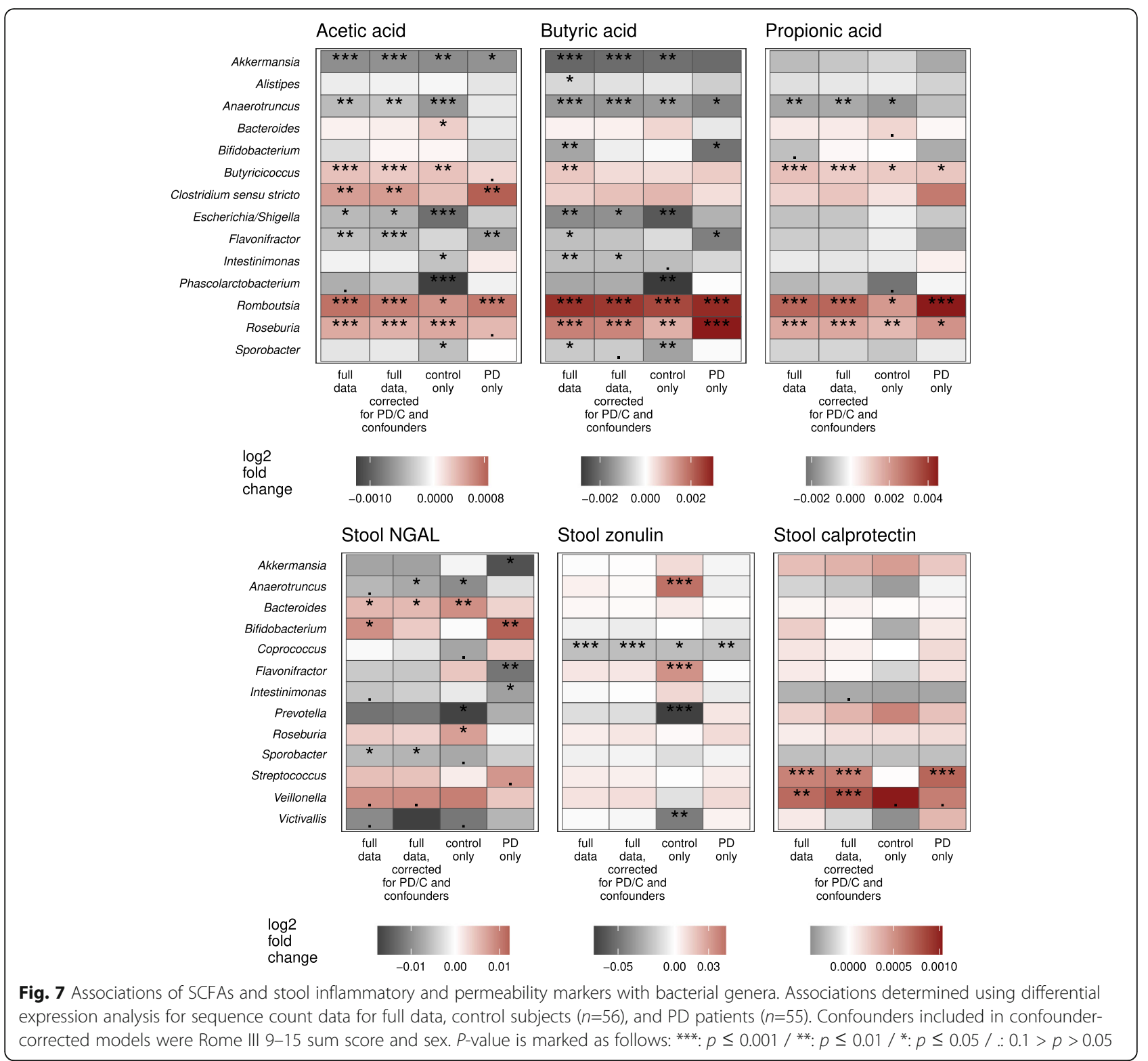

both motor and non-motor PD symptoms even after accounting for disease duration, raising the possibility that higher butyric acid levels could be protective and delay disease onset. In the whole cohort, we also found correlations between stool SCFA levels and non-motor, particularly gastrointestinal and depressive, symptoms, but, with the exception of a positive association between acetic acid and defecation frequency, these correlations were not significant in just the PD subset of the cohort. This could be due to a lack of statistical power or to differences between the control and patient groups in the relative impact of stool SCFAs on the mechanisms driving these symptoms, and it warrants further study.
Though SCFAs are known to have immunomodulatory and barrier-promoting properties, we found no significant correlations between the most abundant stool SCFAs and immune- or permeability-related factors in stool or plasma. While it is possible that SCFA levels may directly impact immunological pathways not assessed in this study, the lack of clear association between SCFA production and inflammation likely evinces the complexity of the relationship between these physiological processes as well as the complexity of immune regulation overall. Both pro- and anti-inflammatory activities of SCFAs have been documented [44, 45], and dietary supplementation of SCFAs produces variable effects on inflammation which appear to be strongly 
influenced by the range of SCFA concentrations involved [46]. Further investigation of the effects of SCFAs on immune responses in the gut, the blood, and the central nervous system in the context of PD pathology is needed.

NGAL, an epithelium-derived antimicrobial glycoprotein overexpressed in inflammatory conditions [47], has been found at higher levels in the plasma of PD patients compared to controls [43]. To the best of our knowledge, this comparison had not been performed in stool, and we found no significant differences in fecal NGAL levels between patients and controls. We also found no significant indications of greater intestinal permeability in this cohort of PD patients as measured by stool zonulin or plasma LBP, though other studies have [21, 24, 27]. On the contrary, we found that higher zonulin levels were associated with less severe clinical manifestations of PD. This could be a reflection of the diversity of motor and non-motor symptom subtypes in PD, a possibility which could be explored in a larger cohort [48]. Longitudinal studies assessing intestinal permeability beginning in the earliest stages of PD would also be beneficial to determine its impact on disease symptoms and progression.

It has been proposed that intestinal inflammation in PD is related to gut permeability and to alterations in microbiota composition and that it can contribute to disease pathogenesis [5]. In this study, we found that levels of fecal NGAL were positively correlated with fecal zonulin, supporting a relationship between this intestinal inflammatory response and permeability. Levels of inflammatory and permeability markers were also associated with the stool microbiota. Inverse associations were found between alpha diversity and levels of stool calprotectin and CXCL8 in PD patients, consistent with the concept that an inflamed gut environment can exert selective pressure on the microbiota. NGAL, IL-2, the stool marker PC, and zonulin were also significantly associated with alterations in beta diversity indicating that gut immune responses and permeability were related to gut microbial composition. Furthermore, stool CXCL8 levels were associated with more pronounced constipation in PD, and stool CXCL8 and IL- $1 \beta$, which were found to be increased in a different cohort of PD patients [29], were negatively correlated with the age of PD motor symptom onset in this study, supporting the involvement of gut inflammation in the development and/ or progression of PD pathology.

A notable finding in this study is that the relationships between gut microbes and inflammatory factors and between microbes and their metabolites such as SCFAs in stool differ between PD patients and controls. In control subjects only, SCFAs were inversely associated with alpha diversity, and SCFA associations with beta diversity were stronger in controls than PD patients. Conversely, levels of butyric acid in stool differed significantly by enterotype only among PD patients. Relationships between SCFAs and individual taxa were largely concordant in both subject groups, including positive correlations between stool SCFAs and well-known SCFA producers like Butyricicoccus and Roseburia and a negative correlation with Akkermansia, which SCFAs are known to inhibit [49]. On the other hand, a positive correlation between SCFAs and relative abundance of Bacteroides was found only in controls while a negative correlation with Bifidobacterium was found only in PD patients.

These discrepancies could reflect differences in microbiota composition between patients and controls; Bacteroides is more abundant in controls, and Bifidobacterium is more abundant in PD patients in this cohort [32], which could increase their functional impacts within their respective microbial communities. This could explain the positive association between SCFAs and Bacteroides, a producer of SCFAs, in controls, but not necessarily the inverse relationship between Bifidobacterium and butyric acid in PD patients. Increases in Bifidobacterium are considered butyrogenic due to crossfeeding of butyrate-producing bacteria [50], but the opposite association was found in our PD cohort, suggesting an altered function or strain profile of the Bifidobacterium genus in the metabolic network of the PD microbiota. Further evidence that Bifidobacterium may not be performing a homeostatic function in PD is its positive correlation with the inflammatory marker NGAL in patients only.

PD patients in this cohort had reduced abundance of Prevotella [32], and significant links between lower levels of fecal zonulin and NGAL and Prevotella abundance and enterotype were observed only in the control group. Visual inspection of the data in Additional Files 10 and 12 suggests that a similar trend in the relationship between NGAL and Prevotella exists in the PD group, and differences in the strength of the association in patients and controls may be influenced by the lower abundance of Prevotella in PD. Whether the relationship between Prevotella and zonulin in PD differs from that in controls is less clear, and, as this taxon is frequently reported to be impacted in PD [19, 31, 32, 51-53], future studies evaluating its influence on intestinal permeability in patients are warranted. Detailed examination of differences in bacterial populations and functions in PD patients and controls may be important in developing the next generation of $\mathrm{PD}$ prevention and therapies, as our findings support a relationship between gut microbial composition and diversity and the severity of PD motor symptoms.

\section{Conclusions}

Our study confirms deficiencies in SCFAs and elevated levels of fecal calprotectin in PD. It also implicates low SCFA levels and gastrointestinal inflammation in the 
development and/or progression of $\mathrm{PD}$, relating levels of butyric acid and CXCL8 and IL-1 $\beta$ in stool to age at disease onset. Moreover, this study provides insights into the relationships among key components of the gut environment and systemic responses. We show that although inflammatory markers in the stool and in circulation were highly correlated within one site, they were not correlated across sites, and that levels of SCFAs and inflammatory and permeability markers in stool were not directly related. It has been established that alterations in the composition of the gut microbiota and intestinal immune responses occur in PD, but our findings suggest that the activities of particular bacteria and the nature of their interactions with the host immune system may be changing as well.

Future longitudinal microbiome studies using multiomics approaches with higher taxonomic resolution combined with detailed pheno- and genotyping of subjects can confirm and expand upon our findings in this initial cohort and provide a thorough understanding of how gut microbes and their metabolites are interacting with the host and impacting the etiology, symptoms, and progression of PD. Studies verifying the impact of the molecules and pathways identified in this study on neurodegenerative processes can produce new targets along the gut-brain axis for more effective disease-modifying treatment of this disorder.

\section{Supplementary Information}

The online version contains supplementary material available at https://doi. org/10.1186/s13024-021-00427-6.

Additional file 1: Supplementary Methods. Extended descriptions of measurements for SCFAs and inflammatory and permeability markers and details of statistical analyses.

Additional file 2: $R$ Markdown. The full $R$ code used for the analyses with output.

Additional file 3: Inflammatory and permeability marker and SCFA concentrations. Table detailing A) levels of all analytes in this study by $\mathrm{PD} /$ control status and $\mathbf{B}$ ) by both PD/control status and sex. P-values reflect Wilcoxon rank sum tests.

Additional file 4: Scatterplots for significantly correlated clinical variables and SCFAs or inflammatory or permeability markers. Scatterplots visualizing relationships of significantly ( $p<0.05$, Pearson) correlated analytes and clinical variables $\mathbf{A}$ ) measured in all subjects and $\mathbf{B}$ ) measured only in PD patients.

Additional file 5: Microbial diversity and PD-related variables. A) Table summarizing $p$-values for comparisons of alpha and beta diversity; B) Scatterplots of significant correlations for alpha diversity and PD-related variables; C) NMDS ordination plots for PD-related variables.

Additional file 6: Linear regression for alpha diversity, inflammatory and permeability markers/SCFAs, and PD/control status. A) Model:

$\log ($ variable) $\sim$ sex + PD/control + Diversity; B) Model: $\log ($ variable $) \sim$ sex $+\mathrm{PD} /$ control * Diversity; C) Stool calprotectin and additional confounders derived from Fig. 3; D) Propionic acid and additional confounders derived from Fig. 3.

Additional file 7: Associations of beta diversity with inflammatory and permeability markers/SCFAs and PD/control status. Permutational multivariate analysis of variance using distance matrices $\mathbf{A}$ ) without interactions, B) with interaction (model: community dissimilarity PD/ control * variable).

Additional file 8: NMDS ordination plots for beta diversity, PD/control status, and select analytes. NMDS ordination plots for the stool markers and SCFAs associated with the most notable beta diversity difference. Variables were split into two categories by median.

Additional file 9: Enterotype comparisons. A) Number of subjects classified to each enterotype in the control and PD groups; B) Comparison of inflammatory and permeability markers/SCFA levels between enterotypes. $P$-values reflect Kruskal-Wallis test and values corrected with Benjamini \& Hochberg false discovery rate method.

Additional file 10: Scatterplots of genera relative abundances by SCFA levels. Scatterplots visualizing significant ( $p<0.05$, Pearson correlation) relationships between SCFAs and relative abundance of most differentially abundant bacterial genera as determined by differential expression analysis for sequence count data.

Additional file 11: Differentially abundant taxa for variables of interest. Table of differential expression analysis for sequence count data results for families and genera that had a multiple comparison corrected $p<$ 0.05 in at least one comparison for $\mathbf{A}$ ) acetic, propionic, and butyric acid or B) stool NGAL, zonulin, and calprotectin. Confounders included in the confounder-corrected models were Rome III 9-15 sum score and sex.

Additional file 12: Scatter plots of genera relative abundances by stool inflammatory and permeability marker levels. Scatterplots visualizing significant ( $p<0.05$, Pearson correlation) relationships between A) NGAL, B) zonulin, and C) calprotectin and relative abundance of most differentially abundant bacterial genera as determined by differential expression analysis for sequence count data.

Additional file 13: Table summarizing all key results of this study.

\section{Abbreviations}

SCFAs: Short-chain fatty acids; PD: Parkinson's disease; CSF: Cerebrospinal fluid; LPS: Lipopolysaccharide; LBP: Lipopolysaccharide-binding protein; CRP: C-reactive protein; TLR4: Toll-like receptor 4; FDR: False discovery rate; PCA: Principal component analysis; BMI: Body mass index; SD: Standard deviation; TIA: Transient ischemic attack; ACE: Angiotensin-converting enzyme; AT1: Angiotensin II type 1; PC: Principal component; NGAL: Neutrophil gelatinase-associated lipocalin; NMSS: Non-Motor Symptoms Scale; GDS15: Geriatric Depression Scale-15; IBS: Irritable bowel syndrome; BPS: Victoria Bowel Performance Scale; UPDRS: Unified Parkinson's Disease Rating Scale

\section{Acknowledgements}

This study was supported in part by the Emory Multiplexed Immunoassay Core (EMIC), which is subsidized by the Emory University School of Medicine and is one of the Emory Integrated Core Facilities.

\section{Authors' contributions}

MGT, FS, PA, LP, PABP, JC, VTEA, and MCH developed and organized the study. VTEA, MCH, PABP, JC, KR, LP, PA, and FS collected samples, data, and measurements. VTEA, MCH, and VH analyzed data. VTEA and $\mathrm{MCH}$ drafted the manuscript. All authors read, reviewed, edited, and approved the final manuscript.

\section{Funding}

This study was funded by the Michael J. Fox Foundation for Parkinson's Research, the Academy of Finland (295724, 310835), the Finnish Medical Foundation, and the Hospital District of Helsinki and Uusimaa (UAK1014004, UAK1014005, TYH2018224). The funding bodies did not contribute to the design of the study, to collection, analysis, or interpretation of data, or to the writing of the manuscript.

\section{Availability of data and materials}

Microbiota data are available at the European Nucleotide Archive (accession number PRJEB27564) (https://www.ebi.ac.uk/ena/browser/view/PRJEB27564). Other data and files utilized in this study are available from the corresponding authors upon reasonable request. The complete $\mathrm{R}$ code for analysis is included as Additional File 2. 


\section{Ethics approval and consent to participate}

This study was conducted in accordance with the Declaration of Helsink and was approved by the ethics committee of the Hospital District of Helsinki and Uusimaa. All participants gave informed consent.

\section{Consent for publication}

Not applicable.

\section{Competing interests}

VTEA, PABP, LP, PA, and FS have patents issued (FI127671B \& US10139408B2) and pending (US16/186,663 \& EP3149205) that are assigned to NeuroBiome Ltd.

FS is founder and CEO of Neurolnnovation Oy and NeuroBiome Ltd., is a member of the scientific advisory board and has received consulting fees and stock options from Axial Biotherapeutics.

MGT is an advisor to INmune Bio, Longevity Biotech, Prevail Therapeutics, and Weston Garfield Foundation. MGT has patents issued (US Pat. Nos. 7144987B1 and 7244823B2) and pending (US20150239951, WO2019067789, 62/901698, see efiling Ack37193677, 62/905747, see efilingAck37274773) for co-invention of DN-TNFs.

\section{Author details}

'DNA Sequencing and Genomics Laboratory, Institute of Biotechnology, University of Helsinki, Viikinkaari 5D, 00790 Helsinki, Finland. ${ }^{2}$ Department of Neurology, Helsinki University Hospital, and Department of Neurological Sciences (Neurology), University of Helsinki, ward K4A, Haartmaninkatu 4, Fl-00290 Helsinki, Finland. ${ }^{3}$ Nell Hodgson Woodruff School of Nursing, Emory University, 1520 Clifton Rd, Atlanta, GA 30322, USA. ${ }^{4}$ Department of Physiology, Emory University School of Medicine, 615 Michael St, Atlanta, GA 30322, USA. ${ }^{5}$ Faculty of Chemistry, Biotechnology and Food Science (KBM), Norwegian University of Life Sciences, 1433 Oslo, Ås, Norway. ${ }^{6}$ Department of Neuroscience and Neurology, Center for Translational Research in Neurodegenerative Disease, University of Florida College of Medicine, 1149 Newell Dr., Gainesville, FL 32611, USA.

\section{Received: 21 August 2020 Accepted: 19 January 2021} Published online: 08 February 2021

\section{References}

1. Boertien JM, Pereira PAB, Aho VTE, Scheperjans F. Increasing comparability and utility of gut microbiome studies in Parkinson's disease: a systematic review. J Park Dis. 2019;9(s2):S297-s312.

2. Bullich C, Keshavarzian A, Garssen J, Kraneveld A, Perez-Pardo P. Gut vibes in Parkinson's disease: the microbiota-gut-brain Axis. Move Disord Clin Pract. 2019;6(8):639-51.

3. Nuzum ND, Loughman A, Szymlek-Gay EA, Hendy A, Teo WP, Macpherson H. Gut microbiota differences between healthy older adults and individuals with Parkinson's disease: a systematic review. Neurosci Biobehav Rev. 2020; 112:227-41.

4. Dinan TG, Cryan JF. Gut instincts: microbiota as a key regulator of brain development, ageing and neurodegeneration. J Physiol. 2017;595(2):489-503.

5. Houser MC, Tansey MG. The gut-brain axis: is intestinal inflammation a silent driver of Parkinson's disease pathogenesis? NPJ Parkinson's Dis. 2017;3:3.

6. Cummings JH, Pomare EW, Branch WJ, Naylor CP, Macfarlane GT. Short chain fatty acids in human large intestine, portal, hepatic and venous blood. Gut. 1987;28(10):1221-7.

7. Kaiko GE, Ryu SH, Koues OI, Collins PL, Solnica-Krezel L, Pearce EJ, et al. The colonic crypt protects stem cells from microbiota-derived metabolites. Cell. 2016;167(4):1137.

8. Roediger WE. Utilization of nutrients by isolated epithelial cells of the rat colon. Gastroenterology. 1982;83(2):424-9.

9. Kelly CJ, Zheng L, Campbell EL, Saeedi B, Scholz CC, Bayless AJ, et al. Crosstalk between microbiota-derived short-chain fatty acids and intestinal epithelial HIF augments tissue barrier function. Cell Host Microbe. 2015; 17(5):662-71.

10. Inan MS, Rasoulpour RJ, Yin L, Hubbard AK, Rosenberg DW, Giardina C. The luminal short-chain fatty acid butyrate modulates NF-kappaB activity in a human colonic epithelial cell line. Gastroenterology. 2000;118(4):724-34.

11. Dalile B, Van Oudenhove L, Vervliet B, Verbeke $K$. The role of short-chain fatty acids in microbiota-gut-brain communication. Nat Rev Gastroenterol Hepatol. 2019;16(8):461-78.
12. Unger MM, Spiegel J, Dillmann KU, Grundmann D, Philippeit H, Burmann J, et al. Short chain fatty acids and gut microbiota differ between patients with Parkinson's disease and age-matched controls. Parkinsonism Relat Disord. 2016:32:66-72.

13. Cirstea MS, Yu AC, Golz E, Sundvick K, Kliger D, Radisavljevic N, et al. Microbiota composition and metabolism are associated with gut function in Parkinson's disease. Movement disorders : official journal of the Movement Disorder Society 2020.

14. McGeer PL, Itagaki S, McGeer EG. Expression of the histocompatibility glycoprotein HLA-DR in neurological disease. Acta Neuropathol. 1988;76(6):550-7.

15. Nagatsu T, Mogi M, Ichinose $H$, Togari A. Changes in cytokines and neurotrophins in Parkinson's disease. J Neural Transm Suppl. 2000;60:277-90.

16. Chen X, Hu Y, Cao Z, Liu Q, Cheng Y. Cerebrospinal fluid inflammatory cytokine aberrations in Alzheimer's disease, Parkinson's Disease and Amyotrophic Lateral Sclerosis: A Systematic Review and Meta-Analysis. Front Immunol. 2018;9:2122.

17. Chen $\mathrm{H}$, O'Reilly EJ, Schwarzschild MA, Ascherio A. Peripheral inflammatory biomarkers and risk of Parkinson's disease. Am J Epidemiol. 2008;167(1):90-5.

18. Qin XY, Zhang SP, Cao C, Loh YP, Cheng Y. Aberrations in peripheral inflammatory cytokine levels in Parkinson disease: a systematic review and meta-analysis. JAMA Neurol. 2016;73(11):1316-24.

19. Lin CH, Chen CC, Chiang HL, Liou JM, Chang CM, Lu TP, et al. Altered gut microbiota and inflammatory cytokine responses in patients with Parkinson's disease. J Neuroinflammation. 2019;16(1):129.

20. Brown GC. The endotoxin hypothesis of neurodegeneration. J Neuroinflammation. 2019;16(1):180.

21. Forsyth CB, Shannon KM, Kordower JH, Voigt RM, Shaikh M, Jaglin JA, et al. Increased intestinal permeability correlates with sigmoid mucosa alphasynuclein staining and endotoxin exposure markers in early Parkinson's disease. PLoS One. 2011;6(12):e28032.

22. Perez-Pardo P, Dodiya HB, Engen PA, Forsyth CB, Huschens AM, Shaikh M, et al. Role of TLR4 in the gut-brain axis in Parkinson's disease: a translational study from men to mice. Gut. 2019;68(5):829-43.

23. van ISCD DP. The intestinal barrier in Parkinson's disease: current state of knowledge. J Park Dis. 2019;9(s2):S323-s9.

24. Schwiertz A, Spiegel J, Dillmann U, Grundmann D, Burmann J, Fassbender K, et al. Fecal markers of intestinal inflammation and intestinal permeability are elevated in Parkinson's disease. Parkinsonism Relat Disord. 2018;50:104-7.

25. Mulak A, Koszewicz M, Panek-Jeziorna M, Koziorowska-Gawron E, Budrewicz S. Fecal Calprotectin as a marker of the gut immune system activation is elevated in Parkinson's disease. Front Neurosci. 2019;13:992.

26. de Waal GM, Engelbrecht L, Davis T, de Villiers WJS, Kell DB, Pretorius E. Correlative light-electron microscopy detects lipopolysaccharide and its association with fibrin fibres in Parkinson's disease, Alzheimer's disease and type 2 diabetes mellitus. Sci Rep. 2018;8(1):16798.

27. Hasegawa S, Goto S, Tsuji H, Okuno T, Asahara T, Nomoto K, et al. Intestinal Dysbiosis and lowered serum lipopolysaccharide-binding protein in Parkinson's disease. PLoS One. 2015;10(11):e0142164.

28. Devos D, Lebouvier T, Lardeux B, Biraud M, Rouaud T, Pouclet H, et al. Colonic inflammation in Parkinson's disease. Neurobiol Dis. 2013;50:42-8.

29. Houser MC, Chang J, Factor SA, Molho ES, Zabetian CP, Hill-Burns EM, et al. Stool immune profiles evince gastrointestinal inflammation in Parkinson's disease. Move Disord. 2018;33(5):793-804.

30. Bjarnason I. The use of fecal Calprotectin in inflammatory bowel disease. Gastroenterol Hepatol. 2017;13(1):53-6.

31. Scheperjans F, Aho V, Pereira PA, Koskinen K, Paulin L, Pekkonen E, et al. Gut microbiota are related to Parkinson's disease and clinical phenotype. Move Disord. 2015;30(3):350-8.

32. Aho VTE, Pereira PAB, Voutilainen $S$, Paulin L, Pekkonen $E$, Auvinen $P$, et al. Gut microbiota in Parkinson's disease: temporal stability and relations to disease progression. EBioMedicine. 2019;44:691-707.

33. Kozich JJ, Westcott SL, Baxter NT, Highlander SK, Schloss PD. Development of a dual-index sequencing strategy and curation pipeline for analyzing amplicon sequence data on the MiSeq Illumina sequencing platform. Appl Environ Microbiol. 2013;79(17):5112-20.

34. Schloss PD, Westcott SL, Ryabin T, Hall JR, Hartmann M, Hollister EB, et al. Introducing mothur: open-source, platform-independent, communitysupported software for describing and comparing microbial communities. Appl Environ Microbiol. 2009;75(23):7537-41.

35. Bork P. Enterotypes: Reference-Based Assignments [Website]. 2016. Available from: http://enterotypes.org/. 
36. Costea PI, Hildebrand F, Arumugam M, Backhed F, Blaser MJ, Bushman FD, et al. Enterotypes in the landscape of gut microbial community composition. Nat Microbiol. 2018;3(1):8-16.

37. R Core Team. R: a language and environment for statistical computing Vienna. Austria: R Foundation for Statistical Computing; 2019.

38. McMurdie PJ, Holmes S. Phyloseq: an R package for reproducible interactive analysis and graphics of microbiome census data. PloS One. 2013;8(4): e61217.

39. Oksanen J, Blanchet FG, Friendly M, Kindt R, Legendre P, McGlinn D, et al. Vegan: community ecology package. 2.5-6 ed. 2019. P. R package.

40. Love Ml, Huber W, Anders S. Moderated estimation of fold change and dispersion for RNA-seq data with DESeq2. Genome Biol. 2014;15(12):550.

41. Ma B, McComb E, Gajer P, Yang H, Humphrys M, Okogbule-Wonodi AC, et al. Microbial biomarkers of intestinal barrier maturation in preterm infants. Front Microbiol. 2018:9:2755.

42. Scherf KA, Lindenau AC, Valentini L, Collado MC, Garcia-Mantrana I, Christensen $\mathrm{M}$, et al. Cofactors of wheat-dependent exercise-induced anaphylaxis do not increase highly individual gliadin absorption in healthy volunteers. Clin Transl Allergy. 2019;9:19.

43. Eidson LN, Kannarkat GT, Barnum CJ, Chang J, Chung J, Caspell-Garcia C, et al. Candidate inflammatory biomarkers display unique relationships with alpha-synuclein and correlate with measures of disease severity in subjects with Parkinson's disease. J Neuroinflammation. 2017;14(1):164

44. Li M, van Esch B, Wagenaar GTM, Garssen J, Folkerts G, Henricks PAJ. Proand anti-inflammatory effects of short chain fatty acids on immune and endothelial cells. Eur J Pharmacol. 2018;831:52-9.

45. Park J, Wang Q, Wu Q, Mao-Draayer Y, Kim CH. Bidirectional regulatory potentials of short-chain fatty acids and their G-protein-coupled receptors in autoimmune neuroinflammation. Sci Rep. 2019;9(1):8837.

46. Bach Knudsen KE, Laerke HN, Hedemann MS, Nielsen TS, Ingerslev AK, Gundelund Nielsen DS, et al. Impact of Diet-Modulated Butyrate Production on Intestinal Barrier Function and Inflammation. Nutrients. 2018:10.

47. Thorsvik S, Bakke I, van Beelen GA, Royset ES, Damas JK, Ostvik AE, et al. Expression of neutrophil gelatinase-associated lipocalin (NGAL) in the gut in Crohn's disease. Cell Tissue Res. 2018;374(2):339-48.

48. Sauerbier A, Jenner P, Todorova A, Chaudhuri KR. Non motor subtypes and Parkinson's disease. Parkinsonism Relat Disord. 2016;22(Suppl 1):S41-6.

49. de la Cuesta-Zuluaga J, Mueller NT, Alvarez-Quintero R, Velasquez-Mejia EP, Sierra JA, Corrales-Agudelo V, et al. Higher Fecal Short-Chain Fatty Acid Levels Are Associated with Gut Microbiome Dysbiosis, Obesity, Hypertension and Cardiometabolic Disease Risk Factors. Nutrients. 2018;11(1):51.

50. Riviere A, Selak M, Lantin D, Leroy F, De Vuyst L. Bifidobacteria and butyrateproducing Colon Bacteria: importance and strategies for their stimulation in the human gut. Front Microbiol. 2016;7:979.

51. Bedarf JR, Hildebrand F, Coelho LP, Sunagawa S, Bahram M, Goeser F, et al. Functional implications of microbial and viral gut metagenome changes in early stage L-DOPA-naive Parkinson's disease patients. Genome Med. 2017; 9(1):39.

52. Petrov VA, Saltykova IV, Zhukova IA, Alifirova VM, Zhukova NG, Dorofeeva YB, et al. Analysis of gut microbiota in patients with Parkinson's disease. Bull Exp Biol Med. 2017;162(6):734-7.

53. Mertsalmi TH, Aho VTE, Pereira PAB, Paulin L, Pekkonen E, Auvinen P, et al. More than constipation - bowel symptoms in Parkinson's disease and their connection to gut microbiota. Eur J Neurol. 2017;24(11):1375-83.

\section{Publisher's Note}

Springer Nature remains neutral with regard to jurisdictional claims in published maps and institutional affiliations.

Ready to submit your research? Choose BMC and benefit from:
- fast, convenient online submission
- thorough peer review by experienced researchers in your field
- rapid publication on acceptance
- support for research data, including large and complex data types
- gold Open Access which fosters wider collaboration and increased citations
- maximum visibility for your research: over 100M website views per year
At BMC, research is always in progress.
Learn more biomedcentral.com/submissions

